\title{
Profiles of virulence genes in enterotoxigenic Escherichia coli isolates from suckling piglets in Serbia1)
}

\author{
JADRANKA ŽUTIĆ, OLIVERA VALČIĆ*, VESNA MILIĆEVIĆ, LJUBIŠA VELJOVIĆ, \\ JASNA KURELJUŠIĆ, DRAGAN BACIĆ**, NEMANJA JEZDIMIROVIĆ
}

\begin{abstract}
Department for Healthcare, Institute of Veterinary Medicine of Serbia, Belgrade
*Department of Physiology and Biochemistry, Faculty of Veterinary Medicine, University of Belgrade

**Department of Infectious Animal Diseases and Diseases of Bees, Faculty of Veterinary Medicine, University of Belgrade
\end{abstract}

Žutić J., Valčić O., Milićević V., Veljović Lj., Kureljušić J., Bacić D., Jezdimirović N.

Profiles of virulence genes in enterotoxigenic Escherichia coli isolates from suckling piglets in Serbia

Summary

A total of 120 Escherichia coli $(E$. coli) strains from suckling piglets with diarrhoea and $30 E$. coli strains from healthy piglets were tested for the presence of fimbrial and enterotoxin virulence genes. Out of the 120 isolates sampled from diarrheic piglets, $81(67.5 \%)$ expressed one or more genes encoding virulence factors. Adhesin genes were detected in $52(43.33 \%)$ out of $120 \mathrm{E}$. coli isolates, and the most common among them was F4 adhesin (33.33\%). Genes encoding $E$. coli toxins were detected in $81(67.5 \%)$ isolates. $E$. coli included in the study carried genes for one or more of the following toxins: STa, STb, LT and EAST1. The astA gene encoding EAST1 was the most prevalent and was identified in $72(60 \%) E$. coli isolates. EAST1 toxin was detected in 5 out of 30 isolates (16.7\%) from healthy piglets. Among the 81 isolates expressing virulence genes, a total of 15 different combinations for fimbrial and toxin genes were found. The most common virulence pattern was $\mathrm{F} 4 / \mathrm{STb} / \mathrm{LT} / \mathrm{EAST} 1$ detected in $23.45 \%$ of $E$. coli strains isolated from suckling piglets with diarrhoea. The results indicate that F4 adhesin and EAST1 toxin are the most common in E. coli isolates sampled from diarrhoeic suckling piglets in Serbia.

Keywords: Escherichia coli, adhesin, enterotoxin, virulence genes, diarrhoea

Diarrhoea in the neonatal period is a major health issue and a permanent challenge to the veterinary profession. At the same time, diarrhoea causes serious economic losses in pig production due to increased morbidity, mortality, treatment costs and poor growth $(9,12,18)$. Escherichia coli $(E$. coli) live in the intestines and are part of the commensal microbiota that plays an important role in the protection of the intestinal tract of animals $(21,26)$. Nevertheless, there are $E$. coli strains which cause dangerous infections in suckling and/or weaned piglets. According to different virulence factors, $E$. coli strains are classified in different pathotypes or categories. Enterotoxigenic E. coli (ETEC) is the main diarrheagenic E. coli pathotype, which causes intestinal infections in newborn pigs (2, $3,5,27)$. Enterotoxigenic $E$. coli strains possess two virulence factors: adhesins and enterotoxins. Both factors are essential for the onset of the disease $(13,17)$.

This research was financially supported by the Ministry of Science and Technological Development of the Republic of Serbia, Project No. TR 20151.
Enterotoxigenic $E$. coli strains responsible for neonatal diarrhoea have a set of fimbriae, such as F4 (K88), F5 (K99), F6 (987P) and F41 (6, 7).

The initial step in the pathogenesis of disease is the attachment of the ETEC strains to the microvilli of the small intestine epithelial cells $(17,18,26)$. Adhesins target specific receptors on the intestinal epithelial cells, thus enabling the transfer of enterotoxins on target cells $(7,16,21)$. Two enterotoxin classes are well known: the high molecular weight heat-labile toxin (LT) and low molecular weight heat-stable a and b toxins (STa and STb) $(6,12,16)$. In addition, a number of ETEC strains also produce enteroaggregative heat-stable enterotoxin 1 (EAST1) $(4,26)$. The same toxin has previously been identified within enteroaggregative $E$. coli strains responsible for diarrhoea in humans (22). Enterotoxins stimulate the intestinal epithelium cells to secrete water and electrolytes into the intestinal lumen, thus reducing absorption, which results in diarrhoea $(14,16)$. 
Severe diarrhoea results in dehydration and acidosis, with a possible lethal outcome $(12,21)$. Important factors leading to diarrhoea in piglets are the presence of enterotoxigenic $E$. coli strains in the small intestine and the absence of antibodies against $E$. coli adhesins in the colostrum of sows. E. coli from the environment colonize newborn piglets by the oral route, thus transferring pathogens to naive animals $(14,17)$. The presence of neonatal colibacillosis is quite common in Serbia. In practice, the diagnosis of this disease is based on clinical signs, the age of infected piglets, isolation of $E$. coli in pure culture and the absence of other bacteria or coccidia.

As the clinical picture is not specific, diarrhoea caused by ETEC strains can only be diagnosed by detecting virulence factors, that is, by determining the pathogenic potential of the E. coli isolate. The aim of this study was to evaluate the presence and prevalence of genes encoding the virulence factors in ETEC strains isolated from newborn piglets with diarrhoea. We believe that these results will offer information useful in the prevention and control of neonatal colibacillosis, especially because there is a lack of reliable data from Serbia.

\section{Material and methods}

Sampling. A total of 120 rectal swabs from piglets with diarrhoea and 30 swabs from healthy piglets aged 1-15 days were taken for analysis. The samples were taken during the morning round of visits by the veterinary service of the farms before treatment. The sampled piglets were housed on 3 different conventional farrow-to-finish pig farms located in three epizootiological areas in the north-west of Serbia. Piglets originated from sows not vaccinated against $E$. coli infections. Sampling was performed at the first signs of diarrhoea. One specimen from one piglet from each litter with diarrhoea was used for bacteriological investigation. On each farm, 40 rectal swabs from diarrhoeic piglets and 10 swabs from healthy piglets were sampled. These farms, with a capacity of 800-1000 sows, were located 50 to $70 \mathrm{~km}$ from Belgrade. After sampling, the swabs were delivered within 2 to 3 hours to the Institute of Veterinary Medicine of Serbia, Belgrade.

Bacterial isolates. Samples were inoculated on MacConkey agar (bioMerieux, France) and incubated aerobically overnight at $37^{\circ} \mathrm{C}$. From each sample, 3 lactose ferment- ing colonies from pure culture were selected for E. coli identification by conventional biochemical procedures (indole, methyl red, Voges-Proskauer and citrate utilization tests). BBL Crystal E/N ID kits (Becton Dickinson, USA) were used as a confirmation test. After the identification of strains, only 1 colony was stored in brain heart infusion broth (HiMedia, India) with $20 \%$ glycerol at $-70^{\circ} \mathrm{C}$ pending further analyses.

Detection of virulence genes by standard PCR and multiplex PCR. DNA was prepared from E. coli strains in the following way (27). The colony of an overnight bacterial culture was suspended in $50 \mu 1$ of water and boiled for 10 minutes, and then cooled on ice for 10 minutes. After brief centrifugation at $13000 \mathrm{~g}$, the supernatant was used as the template for PCR. PCR reactions for F4, F5, F6, F41, LT and EAST1 were performed in a $20 \mu \mathrm{l}$ volume using the PCR Hotstar Master Mix Kit (Qiagen) as well as the primers and protocols provided in Table 1. Ten picomoles of each primer and $2 \mu \mathrm{l}$ of DNA supernatant were added to the reaction and constituted the sample. The PCR reaction for STa and STb was performed with primers (20) as multiplex PCR under the following cycling conditions: 1 cycle of $5 \mathrm{~min}$ at $94^{\circ} \mathrm{C} ; 40$ cycles of $30 \mathrm{sec}$ at $94^{\circ} \mathrm{C}, 60 \mathrm{sec}$ at $55^{\circ} \mathrm{C}$ and 120 sec at $72^{\circ} \mathrm{C}$, followed by final elongation at $72^{\circ} \mathrm{C}$ for $5 \mathrm{~min}$.

A volume of $6 \mu \mathrm{l}$ of the amplified PCR products was analyzed by standard gel electrophoresis $(120 \mathrm{~V}, 30 \mathrm{~mA}$, $60 \mathrm{~min}$.) on $2 \%$ agarose gel stained with ethidium bromide and visualized under ultraviolet light. Primer sequences used for all PCR reactions are given in Table 1.

\section{Results and discussion}

The frequency of virulence factors in $E$. coli strains from suckling piglets with diarrhoea. A total of 120 E. coli strains isolated from suckling piglets with diarrhoea were tested for the presence of genes encoding virulence factors. Out of the $120 \mathrm{E}$. coli isolates tested, $81(67.5 \%)$ strains from the clinical cases expressed 1 or more genes encoding virulence factors. Thirtynine $(32.5 \%)$ isolates were negative and could not be labelled as an ETEC pathotype.

Frequency of adhesin genes. Adhesin genes were detected in $52(43.33 \%)$ E. coli isolates from diarrhoeic piglets. The most common among them was F4, which was detected in $40(33.33 \%)$ E. coli isolates. Other adhesin genes were identified in a smaller number of isolates. The F6 gene was detected in 9 isolates

Tab. 1. PCR primers used for enterotoxin and fimbrial gene detection in Escherichia coli isolates from suckling piglets

\begin{tabular}{|l|l|c|l|}
\hline \multicolumn{1}{|c|}{ Target gene } & \multicolumn{1}{|c|}{ Primer sequence } & Amplified products (bp) & \multicolumn{1}{|c|}{ Reference } \\
\hline LT & TTA CGG CGT TAC TAT CCT CT GGT CTC GGT CAG ATA TGT GA & 275 & Alexa et al. (1997) (1) \\
STa (1,2) & TCT TTC CCC TCT TTA GTC AG ACA GGC CGG ATT ACA ACA AAG & 166 & Osek (2001) (20) \\
STb $(1,2)$ & GCC TAT GCA TCT ACA CAA TC TGA GAA ATG GAC AAT GTC CG & 278 & Osek (2001) (20) \\
EAST1 & CCA TCA ACA CAG TAT ATC CGA GGT CGC GAG TGA CGG CTT TGT & 111 & Yamamoto and Nakazawa (1997) (26) \\
F4 & ATC GGT GGT AGT ATC ACT GC AAC CTG CGA CGT CAA CAA GA & 601 & Ojeniyi et al. (1994) (19) \\
F5 & TGC GAC TAC CAA TGC TTC TG TAT CCA CCA TTA GAC GGA GC & 450 & Ojeniyi et al. (1994) (19) \\
F6 & TCT GCT CTT AAA GCT ACT GG AAC TCC ACC GTT TGT ATC AG & 333 & Ojeniyi et al. (1994) (19) \\
\hline F41 & GCA TCA GCG GCA GTA TCT GTC CCT AGC TCA GTA TTA TCA CCT & 380 & Franck et al. (1998) (8) \\
\hline
\end{tabular}


(7.5\%), 4 of which had the F4/ F6 gene combination. Five isolates $(4.16 \%)$ were positive for $\mathrm{F} 41$, and one of them also had the F5 gene. A total of $3(2.5 \%)$ F5-positive strains were identified. Fimbrial adhesins F4, F5, F6 and F41 were not detected in twenty-nine $(24.16 \%)$ E. coli isolates from diarrhoeic piglets.

The frequency of toxin genes. Genes encoding $E$. coli toxins were detected in $81(67.5 \%)$ out of 120 isolates from diarrhoeic piglets. Strains carried genes for 1 or more of the following toxins: STa, STb, LT and EAST1. The ast $A$ gene encoding EAST1 was the most prevalent gene identified in $72(60 \%)$ strains, followed by genes encoding LT in $42(35 \%)$, and STb in 36 $(30 \%)$, while genes encoding STa were detected in $10(8.3 \%)$ E. coli isolates. All strains with Tab. 2. Combinations of adhesin and toxin genes observed in $81 \mathrm{E}$. coli isolates from piglets with diarrhoea

\begin{tabular}{|c|c|c|c|c|c|c|c|c|}
\hline \multirow{2}{*}{ Toxin gene profile } & \multicolumn{8}{|c|}{ No. of isolates carrying genes for fimbriae } \\
\hline & F4 & F5 & F6 & F41 & $\mathrm{F} 4 / \mathrm{F} 6$ & F5/F41 & None $^{a}$ & Total \\
\hline STb/LT/EAST1 & 19 & & & & & & & 19 \\
\hline LT/EAST1 & 12 & & & & & & & 12 \\
\hline STb/EAST1 & 5 & & & & & & & 5 \\
\hline STb/LT/EAST1 & & & & & 2 & & & 2 \\
\hline STa & & & & & 2 & & & 2 \\
\hline STa & & 2 & & & & & & 2 \\
\hline STa & & & & & & 1 & & 1 \\
\hline STa & & & 3 & & & & & 3 \\
\hline STa/STb/EAST1 & & & 2 & & & & & 2 \\
\hline EAST1 & & & & 3 & & & & 3 \\
\hline STb & & & & 1 & & & & 1 \\
\hline STb/EAST1 & & & & & & & 4 & 4 \\
\hline LT/EAST1 & & & & & & & 6 & 6 \\
\hline STb/LT/EAST1 & & & & & & & 3 & 3 \\
\hline EAST1 & & & & & & & 16 & 16 \\
\hline Total & 36 & 2 & 5 & 4 & 4 & 1 & 29 & 81 \\
\hline
\end{tabular}

Explanation: a - E. coli isolates negative for F4, F5, F6 and F41
LT toxins (42) had genes for EAST1, and 36 strains with STb toxins concurrently showed the EAST1 gene. Both genes encoding STb and LT toxins were detected in 19 F4-positive strains, 2 F4/F6-positive strains and 3 isolates negative for fimbriae. Among the 10 strains carrying the gene for STa toxin, 8 had only the STa gene and 2 showed the STa/STb/EAST1 pattern. Among the 72 EAST1-positive strains, 16 carried the ast $A$ gene as the only virulence gene, while the remaining 56 strains contained additional toxin and/or fimbrial genes. The combinations of adhesin and toxin genes in the E. coli isolates are shown in Table 2.

Out of $120 \mathrm{E}$. coli isolates, $52(43.33 \%)$ possessed genes for fimbriae and toxins. All isolates carrying the F4 fimbrial gene possessed one or more enterotoxin genes. Thirty-eight out of $40 \mathrm{~F} 4$-positive isolates possessed genes for EAST1, 33 carried genes for LT, and 26 for STb. The F6 adhesin was present in 7 strains, including 2 F4/F6-positive strains out of $10 \mathrm{STa}$ positive isolates. In addition, 2 isolates showing F5 adhesin genes encoding STa toxin were identified, as well as 1 isolate with F5/F41 adhesins. Four isolates with F41 adhesins carried genes for individual toxins: 3 isolates for EAST1, and 1 isolate for STb. In general, fimbrial isolates with 1 toxin gene were present in 12 isolates, and 40 isolates contained a number of different toxin genes. Among the 81 isolates with virulence genes, 15 different combinations of genes for fimbriae and toxins were found. The most common virulence pattern was F4/STb/LT/EAST1, present in 19 isolates. In 12 isolates, the combination observed was F4/LT/ EAST1. A less common virulence pattern, present in 5 isolates, was F4/STb/EAST1, while F4/F6/STb/LT/
EAST1 and F4/F6/STa were found in 2 isolates each. Adhesin F5 was found in $2 \mathrm{~F} 5 / \mathrm{ST}$ a isolates and $1 \mathrm{~F} 5 /$ $\mathrm{F} 41 / \mathrm{STa}$ isolate. In total, adhesin F6 was discovered in 5 isolates, 3 of which showed the F6/STa pattern and 2 showed the F6/STa/STb/EAST1 pattern. In addition, 3 isolates had the F41/EAST1 combination, and 1 isolate had the F41/STb combination.

The strains from all three farms showed no significant differences in their profiles of virulence genes. The farms are situated in three bordering epizootiological locations in the north-western part of Serbia. The distance between the farms does not exceed $50 \mathrm{~km}$. That distance could have a significant effect on pathogen transmission between them, since shorter distances result in greater transmission risk.

The frequency of virulence factors in $E$. coli isolates from healthy piglets. A total of $30 \mathrm{E}$. coli isolates from clinically healthy piglets were examined, that is, 10 isolates from each of the 3 farms. E. coli strains positive for the ast $A$ gene were detected on all 3 farms. Three EAST1-positive isolates were found on farms $A$ and $C$, whereas 2 positive isolates were identified on farm B. None of the fimbrial adhesin genes and toxic genes STa, STb, LT were found in $E$. coli isolates from healthy piglets.

Diarrhoea in suckling piglets caused by E. coli strains is a problem which causes significant health and economic losses worldwide. For this reason we decided to publish the results of our research on the prevalence of some virulence factors in $E$. coli isolates from diarrhoeic and non-diarrhoeic piglets at 15 days of age. Among the $120 \mathrm{E}$. coli isolates from diarrhoeic piglets, $81(67.5 \%)$ carried one or more virulence genes. 
E. coli isolates with one or more enterotoxic genes are classified as ETEC. Previous results indicate that the most common among virulence genes were ast $A$ genes, which encode EAST1 enterotoxin in ETEC isolates from suckling piglets with diarrhoea. Thus, in Slovakia the presence of the EAST1 toxin gene was established in 144 out of 220 isolates $(65 \%)$ from diarrhoeic piglets aged up to 14 days (25). In the Czech Republic, EAST1 was present in 277 out of 800 strains (34.6\%) (27). In Korea, 48 out of 116 isolates (41.4\%) were positive for EAST1 (3), and in China the percentage of EAST1 strains was slightly lower (31.5\%) (13). In our studies, we also detected a high prevalence of the ast $A$ gene, which was found in 72 out of $120(60 \%)$ E. coli isolates from diarrhoeic piglets. Similar to results published by Yamamoto and Nakazawa (26), we detected ast $A$ genes in F4-positive strains which concurrently carried genes for LT enterotoxin. We found that all 42 LT-positive strains from diarrhoeic piglets contained the astA gene. Similar results of previous investigations were also reported in the Czech Republic, where 174 out of 180 LT-positive strains $(96.7 \%)$ possessed the ast $A$ gene (27). During our investigation we detected EAST1 toxin in 5 out of 30 isolates $(16.7 \%)$ from healthy pigs. The prevalence of EAST1 in E. coli isolates from nondiarrhoeic piglets was reported as $10.8 \%$ in Canada (18) and 9\% in Mexico (24). In Slovakia, 27\% of isolates from healthy piglets were EAST1-positive (25). As a possible explanation for such a high prevalence of EAST1 in healthy piglets, the authors state asymptomatic infections (25), as well as the possibility that, without the presence of other enterotoxins, EAST1 alone may not be able to cause diarrhoea in piglets $(4,18,24)$. Some authors note a varying connection between the ast $A$ gene and F4 adhesin. For example, in Slovakia $100 \%$ of ast $A$ gene-positive strains were also positive for F4 adhesin (25), in Japan 88.2\% (26), in the Czech Republic 64.6\% (27), and in Uganda 3 out of 4 F4-positive strains carried the EAST1 gene (9). Our results are in agreement with the above, as 38 out of 43 EAST1-positive strains $(88.4 \%)$ carried genes for F4, which indicates a close relationship between EAST 1 and F4 genes in isolates from suckling piglets with diarrhoea in Serbia. On the other hand, in Mexico EAST1 was most often found along with genes encoding F41 (24). Studies on the incidence of STb and LT enterotoxins reveal significant differences between countries. In Switzerland, the prevalence of STb toxin was 90\% (23), in Vietnam 83\% (5), in Slovakia 50\% (25), in Uganda 39\% (9) and in China 20\% (13). Our results show a prevalence of $35 \%$ and $30 \%$ for LT and $\mathrm{STb}$ toxins, respectively. Similar results on the prevalence of LT toxin were obtained in the Czech Republic, where $62.8 \%$ of 277 EAST1-positive isolates carried the gene for LT (27). In Korea, LT toxin was the most common (23\%) in 2010 (11), but 3 years later LT and $\mathrm{STb}$ toxins were found to be present with an equal incidence (31\%) (3). Contrary to the above findings, a number of studies report low incidence $(4.3 \%, 4.7 \%$, and $5.6 \%)$ for LT toxin $(5,12,24)$. The results of Ikwap et al. (9) are interesting, as they show absence of both LT and STa toxins in 18 isolates from suckling piglets with diarrhoea. Among enterotoxins, the least common in the present study was STa toxin, whose genes were determined in 10 isolates, that is $8.3 \%$, which was somewhat more than $3.4 \%$ reported from China (13) for E. coli isolates from suckling piglets with diarrhoea. On the other hand, there are reports of a high prevalence of STa toxin, which amounted to $92.1 \%$ in Vietnam, $80.7 \%$ in Japan, and $37.5 \%$ in Mexico $(5,10$, 24). Previous studies report that fimbrial adhesins are widespread in $E$. coli strains isolated from piglets with diarrhoea. In this study, $33.33 \%$ of all $E$. coli isolates from diseased piglets were positive for the F4 gene, which suggests that adhesin F4 is the most prevalent among the E. coli strains tested in Serbia. These findings are in agreement with other studies from different countries in which ETEC F4-positive strains were isolated from piglets with diarrhoea. Thus, $84.1 \%$ of isolates were F4-positive in the Czech Republic (2), $77 \%$ in Switzerland (23), 46\% in Japan (10), 38\% in Slovakia (25), and $31.9 \%$ in Korea (3).

Compared to our previous results, a smaller incidence of F4 adhesin can be seen: F4 was present in $33.33 \%$ of isolates compared to $80.85 \%$ previously found in piglets up to 30 days of age (28). In accordance with a report by Luppi (14), we ascribe this fact to the less frequent occurrence of F4 strains in younger piglets. However, some reports are contradictory, as they state that F4-positive isolates from diarrhoeic piglets are very rare. $\mathrm{F} 4$ adhesin was found in $3 \%$ of isolates in Mexico (24), and in 3.9\% in China (13). Our results show a markedly lower presence of F5, F6 and F41 adhesins. In some countries, some of them were reported to be dominant, for example, F41 in Mexico (24). In this study, the variability of adherence factors was highest for STa toxin in combination with F5, F6, F4/F6 and F5/F41. Similar combinations of genes encoding adhesins and STa toxin were discovered in other countries - for example F5/STa, F6/STa and F4/F6/STa in Korea and Belgium $(12,15)$, as well as $\mathrm{F} 5 / \mathrm{F} 41 / \mathrm{STa}$ and F6/STa/STb/EAST1 in Slovakia (25). It is interesting that not a single strain with STa toxin was associated with F4 or F41 alone, except when in combination with other adhesins. Three of the 5 F41-positive isolates also possessed the ast $A$ gene, and 1 isolate harboured the genes for $\mathrm{F} 41$ and $\mathrm{STb}$ respectively. Findings on the presence of F5, F6, F18 and F41 reported from Uganda are very interesting, as these adhesins were not detected in any of 18 isolates examined there (9).

The present study shows different profiles of virulence factors in $E$. coli isolates from suckling piglets with diarrhoea in Serbia. The results indicate that the most common genes in ETEC isolates from suckling piglets with diarrhoea were those coding for EAST1 
enterotoxin and, to a lesser degree, for $\mathrm{LT}$ and $\mathrm{STb}$ enterotoxins. Among the colonization factors tested, the most commonly detected were genes for F4 fimbrial adhesin. Our results confirm the presence of virulence factors in 15 different combinations of fimbriae and toxin genes. The most common virulence pattern was $\mathrm{F} 4 / \mathrm{STb} / \mathrm{LT} / \mathrm{EAST} 1$. The research presented here is interesting from the epidemiological point of view and will be useful in prevention programmes, such as the vaccination of sows to protect piglets from ETEC strains in the suckling period.

\section{References}

1. Alexa P., Rychlik I., Nejezchleb A., Hamrik J.: Identification of enterotoxinproducing strains of Escherichia coli by PCR and biological methods. Vet. Med-Czech. 1997, 42, 97-100.

2. Alexa P., Stouracova K., Hamrik J., Rychlik I.: Gene typing of the colonisation factors K88 (F4) in enterotoxigenic Escherichia coli strains isolated from diarrhoeic piglets. Vet. Med-Czech. 2001, 46, 46-49.

3. Byun J. W., Jung B. Y., Kim H. Y., Fairbrother J. M., Lee M. H., Lee W. K.: $\mathrm{O}$-serogroups, virulence genes of pathogenic Escherichia coli and Pulsed-field gel electrophoresis (PFGE) patterns of O149 isolates from diarrhoeic piglets in Korea. Vet. Med- Czech. 2013, 58, 468-476.

4. Choi C., Kwon D., Chae C.: Prevalence of the enteroaggregative Escherichia coli heat-stable enterotoxin 1 gene and its relationship with fimbrial and enterotoxin genes in E. coli isolated from diarrheic piglets. J. Vet. Diag. Invest. 2001, 13, 26-29.

5. Do T. N., Cu P. H., Nguyen H. X., Au T. X., Vu Q. N., Driesen S. J., Townsend $K$. M., Chin J. J., Trott D. J.: Pathotypes and serogroups of enterotoxigenic Escherichia coli isolated from pre-weaning pigs in North Vietnam. J. Med. Microbiol. 2006, 55, 93-99.

6.Dubreuil J. D., Isaacson R. E., Schifferli D. M.: Animal enterotoxigenic Escherichia coli. EcoSal Plus 2016, 7 (1).

7.Francis D. H.: Enterotoxigenic Escherichia coli infection in pigs and its diagnosis. J. Swine Health Prod. 2002, 10, 171-175.

8. Franck S. M., Bosworth B. T., Moon H. W.: Multiplex PCR for enterotoxigenic, attaching and effacing, and Shiga toxin-producing Escherichia coli strains from calves. J. Clin. Microbiol. 1998, 36, 1795-1797.

9. Ikwap K., Larsson J., Jacobson M., Owiny D. O., Nasinyama G. W., Nabukenyan I., Mattisson S., Aspan A., Erume J.: Prevalence of adhesin and toxin genes in E. coli strains isolated from diarrheic and non-diarrheic pigs from smallholder herds in northern and eastern Uganda. BMC Microbiol. 2016, 16, 178, 1-9

10. Katsuda K., Kohmoto M., Kawashima K., Tsunemitsu H.: Frequency of enteropathogen detection in suckling and weaned pigs with diarrhoea in Japan. J. Vet. Diagn. Invest. 2006, 18, 350-354.

11. Kim Y. J., Kim J. H., Hur J., Lee J. H.: Isolation of Escherichia coli from piglets in South Korea with diarrhoea and characteristics of the virulence genes. Can. J. Vet. Res. 2010, 74, 59-64.

12. Kwon D., Kim O., Chae C.: Prevalence of genotypes for fimbriae and enterotoxins and of o serogroups in Escherichia coli isolated from diarrheic piglets in Korea. J. Vet. Diagn. Invest. 1999, 11, 146-151.

13. Liu W., Yuan C., Meng X., Du Y., Gao R., Tang J., Shi D.: Frequency of virulence factors in Escherichia coli isolated from suckling pigs with diarrhoea in China. Vet. J. 2014, 199, 286-289.

14. Luppi A.: Swine enteric colibacillosis: diagnosis, therapy and antimicrobial resistance. Porcine Health Management 2017, 3, 16.

15. Mainil J. G., Daube G., Jacquemin E., Pohl P., Kaekenbeeck A.: Virulence plasmids of enterotoxigenic Escherichia coli isolates from piglets. Vet. Microbiol. 1998, 61, 291-301.

16. Nagy B., Fekete P. Z.: Enterotoxigenic E. coli (ETEC) in farm animals. Vet. Res. BioMed Central. 1999, 30, 259-284.

17. Nagy B., Fekete P. Z.: Enterotoxigenic Escherichia coli in veterinary medicine. Int. J. Med. Microbiol. 2005, 295, 443-454.

18. Ngeleka M., Pritchard J., Appleyard G., Middleton D. M., Fairbrother J. M.: Isolation and association of Escherichia coli AIDA-I/STb, rather than EAST1 pathotype, with diarrhea in piglets and antibiotic sensitivity of isolates. J. Vet. Diagn. Invest. 2003, 15, 242-252

19. Ojeniyi B., Ahrens P., Meyling A.: Detection of fimbrial and toxin genes in Escherichia coli and their prevalence in piglets with diarrhoea. The application of colony hybridization assay, polymerase chain reaction and phenotypic assays. J. Vet. Med. B 1994, 41, 49-59.
20. Osek J.: Multiplex polymerase chain reaction assay for identification of enterotoxigenic Escherichia coli strains. J. Vet. Diagn. Invest. 2001, 13, 308-311.

21.Pereira D. A., Vidotto M. C., Nascimento K. A., Ramos dos Santos A. C., Mechler M. L., Guilherme de Oliveira L.: Virulence factors of Escherichia coli in relation to the importance of vaccination in pigs. Ciência Rural, Santa Maria 2016, 46, 1430-1437.

22. Savarino S. J., Fasano A., Watson J., Martin B. M., Levine M. M., Guandalini S., Guerry P.: Enteroaggregative Escherichia coli heat-stable enterotoxin 1 represents another subfamily of E. coli heat-stable toxin. Proc. Natl. Acad. Sci. USA 1993, 90, 3093-3097.

23. Schneeberger M., Dietz O., Kirchgässner C., Schmitt S.: Aetiologically relevant typing of E. coli isolates from diseased pigs in Switzerland during 2014 and 2015. ARC J. Anim. Vet. Sci. 2017, 3(1): Epub ahead of print.

24. Toledo S., Gomez D., Cruz C., Carreon R., Lopez J., Giono S., Castro A. M. Prevalence of virulence genes in Escherichia coli strains isolated from piglets in the suckling and weaning period in Mexico. J. Med. Microbiol. 2012, 61, 148-156.

25. Vu-Khac H., Holoda E., Pilipcinec E., Blanco M., Blanco J. E., Dahbi G., Mora A., Lopez C., Gonzalez E. A., Blanco J.: Serotypes, virulence genes, intimin types and PFGE profiles of Escherichia coli isolated from piglets with diarrhoea in Slovakia. Vet. J. 2007, 174, 176-187.

26. Yamamoto T., Nakazawa M.: Detection and sequences of the enteroaggregative Escherichia coli heat-stable enterotoxin 1 gene in enterotoxigenic E. coli strains isolated from piglets and calves with diarrhea. J. Clin. Microbiol. 1997, 35, 223-227.

27. Zajacova Z. S., Konstantinova L., Aleksa P.: Detection of virulence factors of Escherichia coli focused on prevalence of EAST1 toxin in stool of diarrheic and non-diarrheic piglets and presence of adhesion involving virulence factors in astA positive strains. Vet. Microbiol. 2012, 154, 369-375.

28. Žutić J., Ašanin J., Mišić D., Jakić-Dimić D., Milić N., Ašanin R., Stojanović D. Žutić M.: Isolation of ETEC strains from piglets with diarrhoea in the neonatal period and their typization based on somatic and fimbrial antigens. Acta Vet. (Belgrade) 2010, 60, 497-506.

Corresponding author: Jadranka Žutić DVM, PhD, Institute of Veterinary Medicine of Serbia, Department for Healthcare, Vojvode Toze 14, 11000 Belgrade, Serbia; e-mail: jadranka.zutic@gmail.com 